\title{
Evidence for high-energy resummation effects in Mueller-Navelet jets at the LHC
}

\author{
B. Ducloué, ${ }^{1}$ L. Szymanowski, ${ }^{2}$ and S. Wallon ${ }^{1,3}$ \\ ${ }^{1}$ LPT, Université Paris-Sud, CNRS, 91405, Orsay, France \\ ${ }^{2}$ National Centre for Nuclear Research (NCBJ), Warsaw, Poland \\ ${ }^{3}$ UPMC Univ. Paris 06, faculté de physique, 4 place Jussieu, 75252 Paris Cedex 05, France
}

\begin{abstract}
The study of the production of two forward jets with a large interval of rapidity at hadron colliders was proposed by Mueller and Navelet as a possible test of the high energy dynamics of QCD. We analyze this process within a complete next-to-leading logarithm framework, supplemented by the use of the Brodsky-Lepage-Mackenzie procedure extended to the perturbative Regge dynamics, to find the optimal renormalization scale. This leads to a very good description of the recent CMS data at LHC for the azimuthal correlations of the jets.
\end{abstract}

PACS numbers: 12.38.Cy, 12.38.Qk, 13.85.Hd

Introduction. Many processes have been proposed as a way to probe the high energy dynamics of QCD, described by the Balitsky-Fadin-Kuraev-Lipatov (BFKL) approach [1]. Among the most promising ones is the production of two forward jets separated by a large interval of rapidity at hadron colliders, proposed by Mueller and Navelet 22. The purpose of the present work is to show that the most recent LHC data extracted by the CMS collaboration for the azimuthal correlations of these jets [3] are well described within this framework.

The description of this process involves two main building blocks: the jet vertex, which describes the transition from an incoming parton to a jet, and the Green's function, which describes the pomeron exchange between the vertices. The first results of a complete next-to-leading logarithmic (NLL) calculation, including the NLL corrections both to the Green's function [4] and to the jet vertex [5], showed that the NLL corrections to the jet vertex have a very large effect, leading to a lower cross section and a much larger azimuthal correlation [6]. It was also observed that the results were very dependent on the choice of the scales, especially the renormalization scale $\mu_{R}$ and the factorization scale $\mu_{F}$. This has been confirmed in a more recent study [7, where we used more realistic kinematic cuts. To reduce this dependency, we apply the physically motivated Brodsky-Lepage-Mackenzie (BLM) procedure 8 to fix the renormalization scale, as it was adapted to the resummed perturbation theory à la BFKL in Refs. 9].

Mueller-Navelet jets. The observables which are of interest are the differential cross section $\mathcal{C}_{0}$

$$
\mathcal{C}_{0}=\frac{\mathrm{d} \sigma}{\mathrm{d}\left|\mathbf{k}_{J, 1}\right| \mathrm{d}\left|\mathbf{k}_{J, 2}\right| \mathrm{d} y_{J, 1} \mathrm{~d} y_{J, 2}},
$$

where $\mathbf{k}_{J, 1}, \mathbf{k}_{J, 2}$ are the transverse momenta of the jets and $y_{J, 1}, y_{J, 2}$ are their rapidities, and the azimuthal correlations [10] of the jets

$$
\frac{\mathcal{C}_{n}}{\mathcal{C}_{0}}=\left\langle\cos \left(n\left(\phi_{J, 1}-\phi_{J, 2}-\pi\right)\right)\right\rangle \equiv\langle\cos (n \varphi)\rangle,
$$

where $\phi_{J, 1}, \phi_{J, 2}$ are the azimuthal angles of the two jets. The relative azimuthal angle $\varphi$ is defined such that $\varphi=0$ corresponds to the back-to-back configuration.

The coefficients $\mathcal{C}_{n}$ can be expressed as

$$
\begin{aligned}
\mathcal{C}_{n}= & \left(4-3 \delta_{n, 0}\right) \int d(\mathrm{P} . \mathrm{S}) f\left(x_{1}\right) f\left(x_{2}\right) E_{n, \nu}\left(\mathbf{k}_{1}\right) E_{n, \nu}^{*}\left(\mathbf{k}_{2}\right) \\
& \times V\left(\mathbf{k}_{1}, x_{1}\right) V\left(\mathbf{k}_{2}, x_{2}\right) \cos \left(n \phi_{J 2}\right) \cos \left(n \phi_{J 1}\right) e^{\omega(n, \nu) Y},
\end{aligned}
$$

where $Y=y_{J, 1}-y_{J, 2}$ and we have defined for brevity the integration over the phase space and over the parameter $\nu$ of conformal weight as

$$
\int d(\mathrm{P} . \mathrm{S})=\int \mathrm{d} \nu \mathrm{d} \phi_{J 1} \mathrm{~d}^{2} \mathbf{k}_{1} \mathrm{~d} x_{1} \mathrm{~d} \phi_{J} \mathrm{~d}^{2} \mathbf{k}_{2} \mathrm{~d} x_{2},
$$

where $\nu$ is integrated from $-\infty$ to $+\infty, x_{1(2)}$ is integrated from 0 to 1 and $\phi_{J 1(2)}$ is integrated from 0 to $2 \pi ; f$ are the usual parton distribution functions (PDFs) and $E_{n, \nu}$ are the LL BFKL eigenfunctions $E_{n, \nu}\left(\mathbf{k}_{i}\right)=$ $\frac{1}{\pi \sqrt{2}}\left(\mathbf{k}_{i}^{2}\right)^{i \nu-\frac{1}{2}} e^{i n \phi_{i}}$. The LL jet vertex reads

$$
V_{\mathrm{a}}^{(0)}(\mathbf{k}, x)=\frac{\alpha_{s}}{\sqrt{2}} \frac{C_{A / F}}{\mathbf{k}^{2}} \delta\left(1-\frac{x_{J}}{x}\right)\left|\mathbf{k}_{J}\right| \delta^{(2)}\left(\mathbf{k}-\mathbf{k}_{J}\right),
$$

where $C_{A}=N_{c}=3$ and $C_{F}=\left(N_{c}^{2}-1\right) /\left(2 N_{c}\right)=4 / 3$ are to be used in the case of incoming gluon and quark respectively. The jet vertex $V$ at NLL accuracy can be written as $V_{\mathrm{a}}(\mathbf{k}, x)=V_{\mathrm{a}}^{(0)}(\mathbf{k}, x)+\alpha_{s} V_{\mathrm{a}}^{(1)}(\mathbf{k}, x)$. The expression of $V_{\mathrm{a}}^{(1)}$, which has been recently reobtained in Ref. [11, can be found in Ref. [6]. Its expression in the limit of small cone jets has been computed in Ref. [12] and used in Refs. 13. It was also rederived within the high energy effective action approach in Refs. 114.

At NLL, the eigenvalue of the BFKL kernel is 15 17.

$$
\omega(n, \nu)=\bar{\alpha}_{s} \chi_{0}\left(|n|, \frac{1}{2}+i \nu\right)+\bar{\alpha}_{s}^{2} \tilde{\chi}_{1}\left(|n|, \frac{1}{2}+i \nu\right),
$$

where $\bar{\alpha}_{s}=N_{c} \alpha_{s} / \pi$,

$$
\chi_{0}(n, \gamma)=2 \psi(1)-\psi\left(\gamma+\frac{n}{2}\right)-\psi\left(1-\gamma+\frac{n}{2}\right),
$$


with $\psi(x)=\Gamma^{\prime}(x) / \Gamma(x)$,

$$
\tilde{\chi}_{1}(n, \gamma)=\chi_{1}(n, \gamma)-\frac{\pi b_{0}}{N_{c}} \chi_{0}(n, \gamma) \ln \frac{\left|\mathbf{k}_{J, 1}\right| \cdot\left|\mathbf{k}_{J, 2}\right|}{\mu_{R}^{2}},
$$

where the expression for $\chi_{1}$, which was obtained in Refs. [15], can be found in Eq. (2.17) of Ref. [7].

BLM scale setting. The BLM procedure is a way of absorbing the non conformal terms of the perturbative series in a redefinition of the coupling constant, to improve the convergence of the perturbative series [18]. In practice, one should extract the $\beta_{0}$-dependent part of an observable and choose the renormalization scale to make it vanish. The BLM procedure was first applied to BFKL dynamics in Refs. [9] for the $\gamma^{*} \gamma^{*}$ total crosssection, considering the NLL corrections to the Green's function but using the LL $\gamma^{*}$ impact factor, with the important outcome of stabilizing the NLL BFKL intercept. This method was used in a similar spirit in Refs. [19].
We follow the same line of thought, taking into account the NLL corrections to the jet vertex.

In the expression of the coefficients $\mathcal{C}_{n}$, the renormalization scale $\mu_{R}$ enters both $\omega$ (through $\alpha_{s}$ and the second term of $\tilde{\chi}_{1}$ which carries an explicit dependence on $\mu_{R}$ ) and $V$. To separate the parts which depend on $\mu_{R}$ from those which do not, we rewrite Eq. (3) as

$$
\begin{aligned}
\mathcal{C}_{n}= & \alpha_{s}^{2}\left(4-3 \delta_{n, 0}\right) \int d(\mathrm{P} . \mathrm{S}) D\left(\mathbf{k}_{1}, x_{1}\right) D\left(\mathbf{k}_{2}, x_{2}\right) \\
& \times A\left(x_{1}, \mathbf{k}_{1}, \phi_{J 1}\right) A^{*}\left(x_{2}, \mathbf{k}_{2}, \phi_{J 2}\right) e^{\omega(n, \nu) Y},
\end{aligned}
$$

where $A\left(x_{i}, \mathbf{k}_{i}, \phi_{J i}\right)=f\left(x_{i}\right) E_{n, \nu}\left(\mathbf{k}_{i}\right) \cos \left(n \phi_{J i}\right)$. As $V^{(0)}$ and $V^{(1)}$ both contain a global $\alpha_{s}$ factor, we have defined $D^{(i)}(\mathbf{k}, x)=V^{(i)}(\mathbf{k}, x) / \alpha_{s}$ to make $D^{(0)}$ and $D^{(1)} \alpha_{s^{-}}$ independent. We now focus on the $\mu_{R}$-dependent part $D\left(\mathbf{k}_{1}, x_{1}\right) D\left(\mathbf{k}_{2}, x_{2}\right) e^{\omega(n, \nu) Y} \equiv B_{n}$ of Eq. (9). It can be expanded as the following series at NLL accuracy, for an arbitrary renormalization scale $\mu_{R \text {,init }}$,

$$
\begin{aligned}
B_{n}= & {\left[D^{(0)}\left(\mathbf{k}_{1}, x_{1}\right) D^{(0)}\left(\mathbf{k}_{2}, x_{2}\right)+\alpha_{s}\left(\mu_{R, \text { init }}\right)\left(D^{(1)}\left(\mathbf{k}_{1}, x_{1}\right) D^{(0)}\left(\mathbf{k}_{2}, x_{2}\right)+D^{(0)}\left(\mathbf{k}_{1}, x_{1}\right) D^{(1)}\left(\mathbf{k}_{2}, x_{2}\right)\right)\right] } \\
& \times \sum_{m=0}^{\infty} \frac{\left(\bar{\alpha}_{s}\left(\mu_{R, \text { init }}\right) \chi_{0}(n, \gamma) Y\right)^{m}}{m !}\left(1+m \bar{\alpha}_{s}\left(\mu_{R, \text { init }}\right) \frac{\tilde{\chi}_{1}(n, \gamma}{\chi_{0}(n, \gamma}\right) .
\end{aligned}
$$

Up to now, all the quantities we introduced were defined in the $\overline{\mathrm{MS}}$ scheme. However, the BLM procedure is more conveniently applied in a physical renormalization scheme, so we first perform the transition from the $\overline{\mathrm{MS}}$ to the MOM scheme, which is equivalent to writing [20]

$$
\alpha_{\overline{\mathrm{MS}}}=\alpha_{\mathrm{MOM}}\left(1+\alpha_{\mathrm{MOM}} \frac{T_{\mathrm{MOM}}}{\pi}\right)
$$

where $T_{\mathrm{MOM}}=T_{\mathrm{MOM}}^{\beta}+T_{\mathrm{MOM}}^{\text {conf }}$,

$T_{\mathrm{MOM}}^{\text {conf }}=\frac{N_{c}}{8}\left[\frac{17}{2} I+\frac{3}{2}(I-1) \xi+\left(1-\frac{1}{3} I\right) \xi^{2}-\frac{1}{6} \xi^{3}\right]$,

$T_{\mathrm{MOM}}^{\beta}=-\frac{\beta_{0}}{2}\left(1+\frac{2}{3} I\right)$,

where $\beta_{0}=\left(11 N_{c}-2 N_{f}\right) / 3, N_{f}$ is the number of flavors, $I=-2 \int_{0}^{1} d x \ln (x) /\left[x^{2}-x+1\right] \simeq 2.3439$ and $\xi$ is a gauge parameter. The variation of $B_{n}$ when going from the $\overline{\mathrm{MS}}$ to the MOM scheme is

$$
\begin{aligned}
\delta B_{n}= & D^{(0)}\left(\mathbf{k}_{1}, x_{1}\right) D^{(0)}\left(\mathbf{k}_{2}, x_{2}\right) \bar{\alpha}_{s}\left(\mu_{R, \text { init }}\right) \frac{T_{\mathrm{MOM}}}{N_{c}} \\
& \times \sum_{m=1}^{\infty} \frac{\left(\bar{\alpha}_{s}\left(\mu_{R, \text { init }}\right) \chi_{0}(n, \gamma) Y\right)^{m}}{(m-1) !}
\end{aligned}
$$

so that $B_{n, \mathrm{MOM}}=B_{n}+\delta B_{n}$. To express $B_{n, \mathrm{MOM}}$ as a function of an arbitrary renormalization scale $\mu_{R}$ we write $\alpha\left(\mu_{R, \text { init }}\right)$ as

$$
\alpha_{s}\left(\mu_{R, \text { init }}\right)=\alpha_{s}\left(\mu_{R}\right)\left(1-\alpha_{s}\left(\mu_{R}\right) \frac{\beta_{0}}{4 \pi} \ln \frac{\mu_{R, \text { init }}^{2}}{\mu_{R}^{2}}\right) .
$$

We shall now insert Eq. (14) in the expression of $B_{n, \mathrm{MOM}}$ and extract the $\beta_{0}$-dependent part. One can see from the expression of $V_{\mathrm{a}}^{(1)}$ given in [6] that the term which depends on $\beta_{0}$ is proportional to the leading order part of the vertex, i.e. $D^{(1) \beta}\left(\mathbf{k}_{i}, x_{i}\right)=-\frac{\beta_{0}}{2 \pi} \ln \frac{\mathbf{k}_{i}}{\mu_{R, \text { init }}} D^{(0)}\left(\mathbf{k}_{i}, x_{i}\right)$. Thus the part of $B_{n, \mathrm{MOM}}$ proportional to $\beta_{0}$ reads 


$$
\begin{aligned}
B_{n, \mathrm{MOM}}^{\beta}= & D^{(0)}\left(\mathbf{k}_{1}, x_{1}\right) D^{(0)}\left(\mathbf{k}_{2}, x_{2}\right) \sum_{m=0}^{\infty} \alpha_{s}\left(\mu_{R}\right)^{m+1} \chi_{0}(n, \gamma)^{m}\left(\frac{Y N_{c}}{\pi}\right)^{m} \frac{1}{m !}\left[-\frac{\beta_{0}}{2 \pi} \ln \frac{\left|\mathbf{k}_{1}\right| \cdot\left|\mathbf{k}_{2}\right|}{\mu_{R, \text { init }}^{2}}\right. \\
& \left.+m \frac{N_{c}}{\pi}\left(\frac{\tilde{\chi}_{1}^{\beta}(n, \gamma)}{\chi_{0}(n, \gamma)}+\frac{T_{\mathrm{MOM}}^{\beta}}{N_{c}}\right)-m \frac{\beta_{0}}{4 \pi} \ln \frac{\mu_{R, \text { init }}^{2}}{\mu_{R}^{2}}\right]
\end{aligned}
$$

where $\tilde{\chi}_{1}^{\beta}$ and $T_{\mathrm{MOM}}^{\beta}$ are the $\beta_{0}$-dependent parts of $\tilde{\chi}_{1}$ and $T_{\mathrm{MOM}}$ respectively. The optimal scale $\mu_{R, \mathrm{BLM}}$ is the value of $\mu_{R}$ that makes the expression inside the brackets vanish. Taking into account the fact that our initial scale is $\mu_{R \text {,init }}=\sqrt{\left|\mathbf{k}_{J, 1}\right| \cdot\left|\mathbf{k}_{J, 2}\right|}$ and that $D^{(0)}\left(\mathbf{k}_{i}, x_{i}\right)$ contains a factor $\delta^{(2)}\left(\mathbf{k}_{i}-\mathbf{k}_{J, i}\right)$ which will enforce $\left|\mathbf{k}_{i}\right|=\left|\mathbf{k}_{J, i}\right|$ after integrating over $\mathrm{d}^{2} \mathbf{k}_{i}$, we need to solve the equation

$$
\frac{N_{c}}{\pi}\left(\frac{\chi_{1}^{\beta}(n, \gamma)}{\chi_{0}(n, \gamma)}+\frac{T_{\mathrm{MOM}}^{\beta}}{N_{c}}\right)-\frac{\beta_{0}}{4 \pi} \ln \frac{\left|\mathbf{k}_{J, 1}\right| \cdot\left|\mathbf{k}_{J, 2}\right|}{\mu_{R, \mathrm{BLM}}^{2}}=0,
$$

whose solution is

$\mu_{R, \text { BLM }}^{2}=\left|\mathbf{k}_{J, 1}\right| \cdot\left|\mathbf{k}_{J, 2}\right| \exp \left[\frac{1}{2} \chi_{0}(n, \gamma)-\frac{5}{3}+2\left(1+\frac{2}{3} I\right)\right]$.

Theoretical uncertainties. Despite the fact that we have used the BLM procedure to fix the renormalization scale, several theoretical uncertainties remain.

First, the scale of the prefactor $\alpha_{s}^{2}$ in Eq. 9 is not fixed in our implementation of the scale fixing procedure. To evaluate the corresponding uncertainty, we consider two cases, namely either we take for this scale $\mu_{R, \text { BLM }}$ or $\mu_{R, \text { init }}$.

Second, our calculation involves the factorization scale $\mu_{F}$ which enters both the PDFs and the hard part. In principle, one should vary independently $\mu_{R}$ and $\mu_{F}$. But since the choice $\mu_{R}=\mu_{F}$ is made by all PDFs fitting collaborations we are aware of, one could argue that, for consistency, we should do the same. To estimate the reliability of our results, we did two evaluations: one with $\mu_{F}=\mu_{R}=\mu_{R, \text { BLM }}$ and one with the 'natural' choice $\mu_{F}=\sqrt{\left|\mathbf{k}_{J, 1}\right| \cdot\left|\mathbf{k}_{J, 2}\right|}$. In both cases, we chose the single scale entering the PDFs as $\mu_{F}$.

Third, several methods 21, 22] have been proposed to improve the NLL BFKL Green's function by imposing its compatibility with DGLAP 23] in the collinear limit. As in Ref. [7, we implemented scheme 3 of Ref. [21] and found that the effect of such collinear improvement was important for the cross section but much smaller than the two previous uncertainties for all the angular quantities we study here.

Results. Recently the CMS collaboration measured the azimuthal decorrelation of Mueller-Navelet jets at the LHC at a center of mass energy of $7 \mathrm{TeV}[3$. We here compare our results using the BLM procedure to this measurement. The quantities we discussed in the previous sections were differential with respect to the trans- verse momenta $\mathbf{k}_{J, 1}, \mathbf{k}_{J, 2}$ and the rapidities $y_{J, 1}, y_{J, 2}$ of the jets. Here we try to stay as close as possible to the configuration used in Ref. [3]: $y_{J, 1}$ and $y_{J, 2}$ run between 0 and 4.7 and we integrate $\mathbf{k}_{J, 1}$ and $\mathbf{k}_{J, 2}$ from $35 \mathrm{GeV}$ to $60 \mathrm{GeV}$. The CMS collaboration did not use an upper cut on the transverse momenta of the jets, but we have to do so for numerical reasons. We have checked that our results do not depend strongly on the value of this cut, as the cross section is quickly decreasing with increasing transverse momenta. We use the anti- $k_{t}$ jet algorithm 24] with a size parameter $R=0.5$ and the MSTW 2008 PDFs [25]. The results displayed in every figure include the NLL BFKL calculation with the 'natural' choice $\mu_{R}=\mu_{F}=\sqrt{\left|\mathbf{k}_{J, 1}\right| \cdot\left|\mathbf{k}_{J, 2}\right|}$ (dashed line), the NLL BFKL calculation with the BLM scale choice (gray uncertainty band) and the CMS data (dots with error bars). In our uncertainty band, we include the three effects discussed in the previous section.

Before comparing our results with data, we would like to note that our calculation is performed at the partonic level and does not include hadronization effects. However the magnitude of these effects was estimated in [3] to be smaller than the experimental uncertainties, which justifies this comparison. We also did not take into account multi-parton interactions, in which several partons from the same hadron take part in the interaction, as there is for now no theoretical framework to deal with such contributions at small $x$.

We first show results for the angular correlations $\langle\cos \varphi\rangle,\langle\cos 2 \varphi\rangle$ and $\langle\cos 3 \varphi\rangle$ as a function of the relative rapidity $Y=y_{J, 1}-y_{J, 2}$ on Figs. 1, 2 and 3 , respectively. The conclusion for these three observables is similar: when one uses the 'natural' scale, the NLL BFKL calculation is always above the data. But these data are much better described when setting the scale according to the BLM procedure.

On the other hand, the ratios $\langle\cos 2 \varphi\rangle /\langle\cos \varphi\rangle$ and $\langle\cos 3 \varphi\rangle /\langle\cos 2 \varphi\rangle$ are almost not affected by the BLM procedure (see Figs. 4 and 5). This is because these observables are very stable with respect to the scales, as was noticed before in Refs. 6, 7, 17.

Another interesting observable measured in Ref. 3 is the azimuthal distribution of the jets $\frac{1}{\sigma} \frac{d \sigma}{d \varphi}$, which can be expressed as

$$
\frac{1}{\sigma} \frac{d \sigma}{d \varphi}=\frac{1}{2 \pi}\left\{1+2 \sum_{n=1}^{\infty} \cos (n \varphi)\langle\cos (n \varphi)\rangle\right\} .
$$




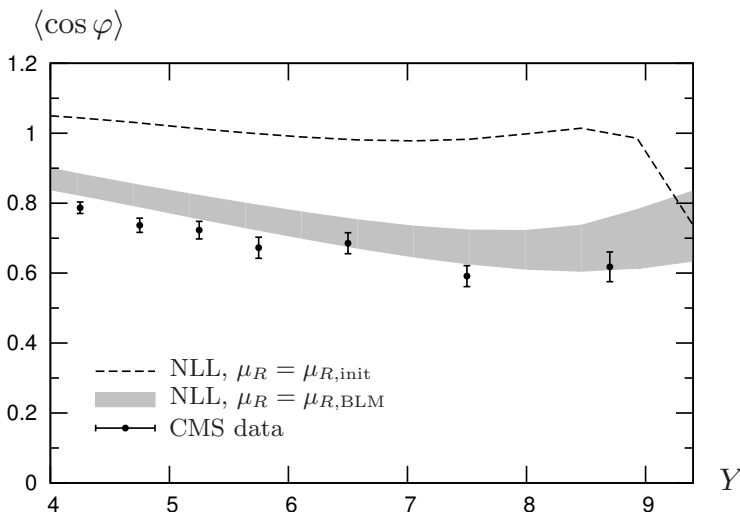

FIG. 1. Variation of $\langle\cos \varphi\rangle$ as a function of $Y$ at NLL accuracy compared with CMS data.

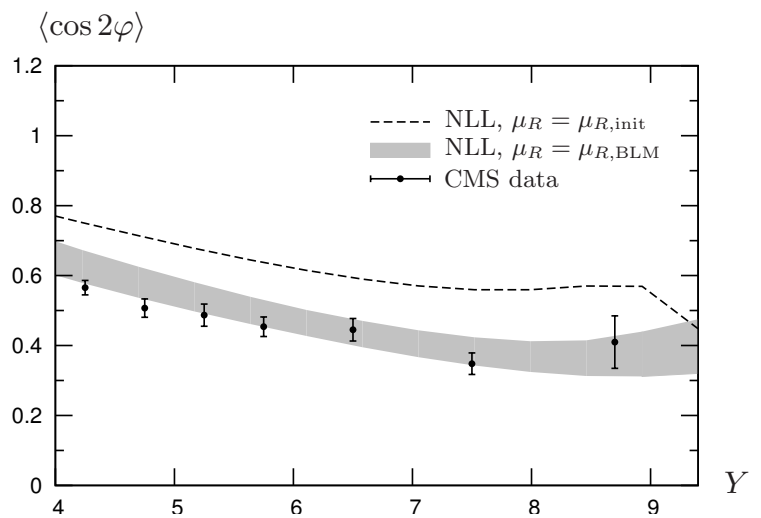

FIG. 2. Variation of $\langle\cos 2 \varphi\rangle$ as a function of $Y$ at NLL accuracy compared with CMS data.

In Fig. 6 we show the comparison of our calculation with the data for the azimuthal distribution integrated over the range $6.0<Y<9.4$. We observe that using the 'natural' scale $\mu=\sqrt{\left|\mathbf{k}_{J, 1}\right| \cdot\left|\mathbf{k}_{J, 2}\right|}$, the BFKL calculation is slightly above the data for $\varphi \lesssim 1$ and then becomes much lower than the data, even reaching negative values

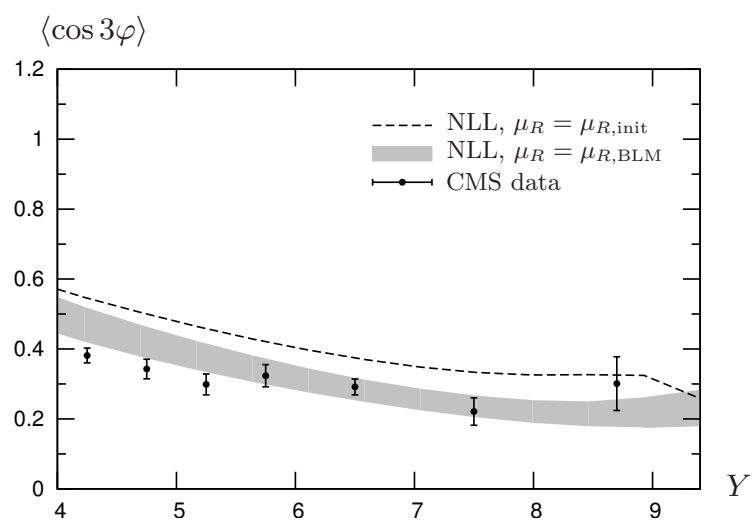

FIG. 3. Variation of $\langle\cos 3 \varphi\rangle$ as a function of $Y$ at NLL accuracy compared with CMS data.

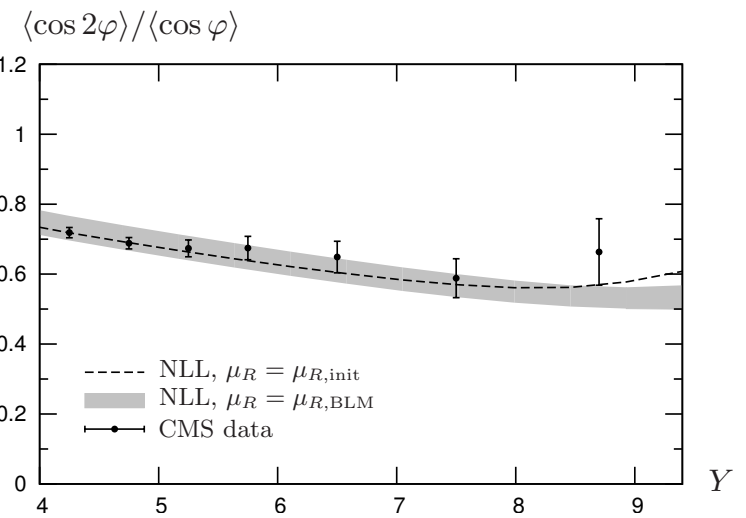

FIG. 4. Variation of $\langle\cos 2 \varphi\rangle /\langle\cos \varphi\rangle$ as a function of $Y$ at NLL accuracy compared with CMS data.

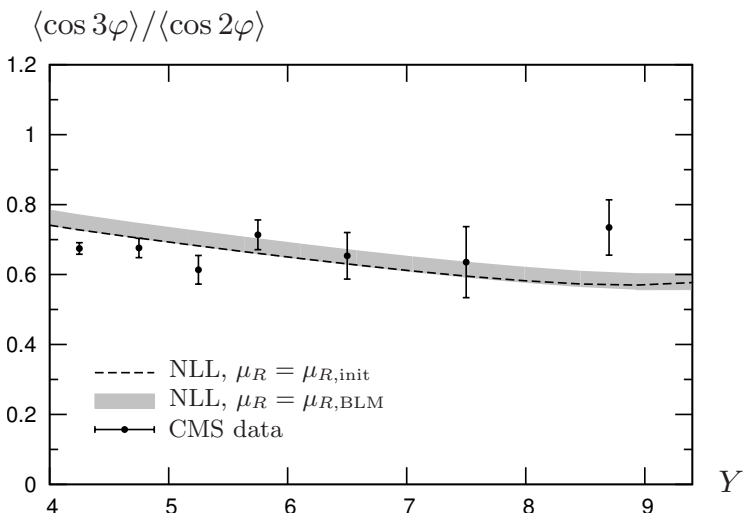

FIG. 5. Variation of $\langle\cos 3 \varphi\rangle /\langle\cos 2 \varphi\rangle$ as a function of $Y$ at NLL accuracy compared with CMS data.

for $\varphi \sim \pi$. This issue does not arise when using BLM and the agreement with data then becomes very good over the full $\varphi$ range.

Comparison with fixed-order. Since the CMS collaboration considered configurations with identical lower cuts on the jets transverse momenta, which would lead to unreliable results in a fixed-order treatment [26], a direct comparison of our analysis with this approach cannot be performed. In Fig. 7 we show the comparison of our BFKL calculation with the results obtained with the NLO fixed-order code Dijet 27 for the ratio $\langle\cos 2 \varphi\rangle /\langle\cos \varphi\rangle$ in the same kinematics as for previous results, but with the requirement that at least one jet has a transverse momentum larger than $50 \mathrm{GeV}$. As in [7, we see that there is a clear difference between BFKL and fixed-order so we expect that an experimental analysis in an asymmetric configuration would discriminate between these approaches.

Energy-momentum conservation. A general weakness of BFKL calculations is the absence of strict energymomentum conservation. This has been studied for Mueller-Navelet jets in the past [28, 29], using the leading order jet vertex. These studies showed that this is 


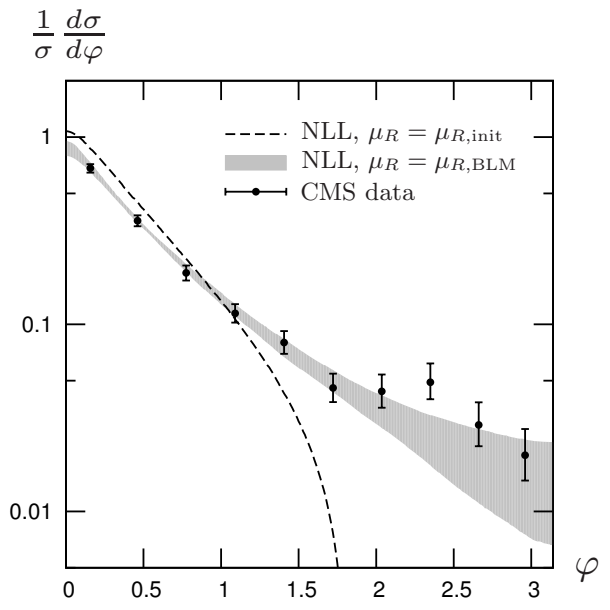

FIG. 6. Azimuthal distribution at NLL accuracy compared with CMS data.

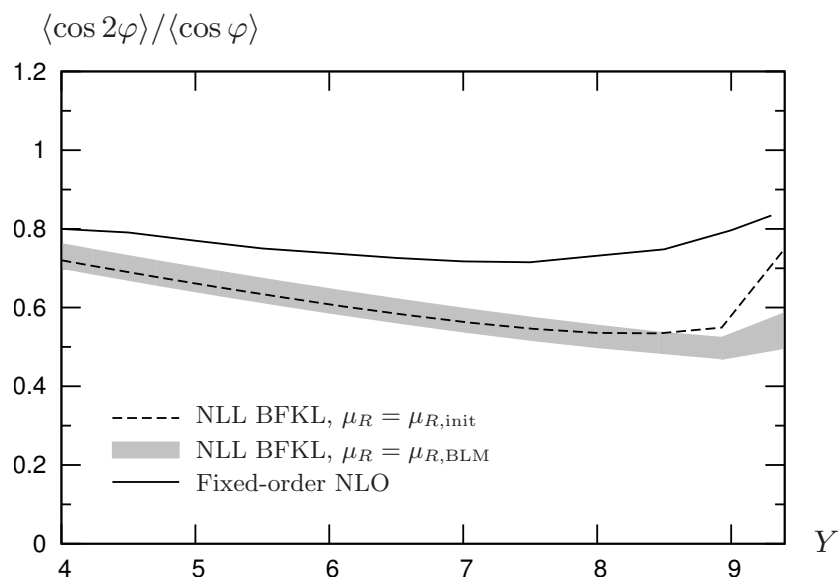

FIG. 7. Variation of $\langle\cos 2 \varphi\rangle /\langle\cos \varphi\rangle$ as a function of $Y$ at NLL accuracy compared with a fixed order treatment.

mainly an issue when $\mathbf{k}_{J, 1}$ and $\mathbf{k}_{J, 2}$ are different. This effect should not be dramatic here, as we use the same lower cut on these variables when comparing with CMS data and the cross section decreases quickly with increasing $\mathbf{k}_{J, 1}, \mathbf{k}_{J, 2}$. Also we expect that the inclusion of the NLL corrections to the jet vertex improves the situation.

Conclusions. In this work we have studied the azimuthal correlations of Mueller-Navelet jets and compared the predictions of a full NLL BFKL calculation with data taken at the LHC. We have shown that using the BLM procedure to fix the renormalization scale leads to a very good agreement with the data, much better than when using the 'natural' value $\sqrt{\left|\mathbf{k}_{J, 1}\right| \cdot\left|\mathbf{k}_{J, 2}\right|}$.

We thank François Gelis and Edmond Iancu for warm hospitality at IPhT Saclay. We thank Michel Fontannaz, Grzegorz Brona, Hannes Jung, Victor Kim and the Low-x 2013 Workshop participants for stimulating discussions.

This work is supported by the French Grant PEPS-PTI, the Polish Grant NCN No. DEC2011/01/B/ST2/03915 and the Joint Research Activity
Study of Strongly Interacting Matter (HadronPhysics3, Grant Agreement n.283286) under the 7th Framework Programme of the European Community.

[1] V. S. Fadin, E. A. Kuraev, and L. N. Lipatov, Phys. Lett. B60, 50 (1975)E. A. Kuraev, L. N. Lipatov, and V. S. Fadin, Sov. Phys. JETP 44, 443 (1976)45, 199 (1977)I. I. Balitsky and L. N. Lipatov, Sov. J. Nucl. Phys. 28, 822 (1978)

[2] A. H. Mueller and H. Navelet, Nucl. Phys. B282, 727 (1987)

[3] CMS Collaboration, Azimuthal angle decorrelations of jets widely separated in rapidity in pp collisions at $\sqrt{s}=7$ TeV, CMS Physics Analysis Summary CMS-PAS-FSQ12-002 (2013) http://cds. cern.ch/record/1547075

[4] V. S. Fadin and L. N. Lipatov, Phys. Lett. B429, 127 (1998), hep-ph/9802290M. Ciafaloni and G. Camici, B430, 349 (1998), hep-ph/9803389

[5] J. Bartels, D. Colferai, and G. P. Vacca, Eur. Phys. J. C24, 83 (2002), hep-ph/0112283C29, 235 (2003), hep$\mathrm{ph} / 0206290$

[6] D. Colferai, F. Schwennsen, L. Szymanowski, and S. Wallon, JHEP 12, 026 (2010), arXiv:1002.1365 [hep-ph]

[7] B. Ducloué, L. Szymanowski, and S. Wallon, JHEP 1305, 096 (2013), arXiv:1302.7012 [hep-ph]

[8] S. J. Brodsky, G. P. Lepage, and P. B. Mackenzie, Phys. Rev. D28, 228 (1983)

[9] S. J. Brodsky, V. S. Fadin, V. T. Kim, L. N. Lipatov, and G. B. Pivovarov, JETP Lett. 70, 155 (1999), hepph/990122976, 249 (2002), hep-ph/0207297

[10] V. Del Duca and C. R. Schmidt, Phys. Rev. D49, 4510 (1994), hep-ph/9311290W. J. Stirling, Nucl. Phys. B423, 56 (1994), hep-ph/9401266

[11] F. Caporale, D. Y. Ivanov, B. Murdaca, A. Papa, and A. Perri, JHEP 1202, 101 (2012), arXiv:1112.3752 [hep$\mathrm{ph}]$

[12] D. Y. Ivanov and A. Papa, JHEP 1205, 086 (2012), arXiv:1202.1082 [hep-ph]

[13] F. Caporale, D. Y. Ivanov, B. Murdaca, and A. Papa(2012), arXiv:1211.7225 [hep-ph]F. Caporale, B. Murdaca, A. Sabio Vera, and C. Salas, Nucl. Phys. B875, 134 (2013), arXiv:1305.4620 [hep-ph]

[14] M. Hentschinski and A. Sabio Vera, Phys. Rev. D85, 056006 (2012), arXiv:1110.6741 [hep-ph]G. Chachamis, M. Hentschinski, J. D. Madrigal, and A. Sabio Vera, D87, 076009 (2013), arXiv:1212.4992 [hep-ph]

[15] A. V. Kotikov and L. N. Lipatov, Nucl. Phys. B582, 19 (2000), hep-ph/000400\& Nucl. Phys. B661, 19 (2003), hep-ph/0208220

[16] D. Y. Ivanov and A. Papa, Nucl. Phys. B732, 183 (2006), hep-ph/0508162

[17] A. Sabio Vera, Nucl. Phys. B746, 1 (2006), hep$\mathrm{ph} / 0602250 \mathrm{~A}$. Sabio Vera and F. Schwennsen, B776, 170 (2007), hep-ph/0702158F. Schwennsen(2007), hep$\mathrm{ph} / 0703198$

[18] The BLM procedure was later extended to all orders, leading to the principle of maximal conformality (PMC) 30.

[19] M. Angioni, G. Chachamis, J. Madrigal, and A. Sabio Vera, Phys. Rev. Lett. 107, 191601 (2011), 
arXiv:1106.6172 [hep-th] M. Hentschinski, A. Sabio Vera, and C. Salas, 110, 041601 (2013), arXiv:1209.1353 [hepph]Phys. Rev. D87, 076005 (2013), arXiv:1301.5283 [hep-ph]

[20] W. Celmaster and R. J. Gonsalves, Phys. Rev. Lett. 42, 1435 (1979) Phys. Rev. D20, 1420 (1979)

[21] G. P. Salam, JHEP 07, 019 (1998), hep-ph/9806482

[22] M. Ciafaloni and D. Colferai, Phys. Lett. B452, 372 (1999), hep-ph/9812366.M. Ciafaloni, D. Colferai, and G. P. Salam, Phys. Rev. D60, 114036 (1999), hepph/9905566M. Ciafaloni, D. Colferai, G. P. Salam, and A. M. Stasto, D68, 114003 (2003), hep-ph/0307188

[23] V. N. Gribov and L. N. Lipatov, Sov. J. Nucl. Phys. 15, 438 (1972)L. N. Lipatov, 20, 94 (1975)G. Altarelli and G. Parisi, Nucl. Phys. B126, 298 (1977)Y. L. Dokshitzer, Sov. Phys. JETP 46, 641 (1977)

[24] M. Cacciari, G. P. Salam, and G. Soyez, JHEP 0804, 063 (2008), arXiv:0802.1189 [hep-ph]

[25] A. D. Martin, W. J. Stirling, R. S. Thorne, and G. Watt, Eur. Phys. J. C63, 189 (2009), arXiv:0901.0002 [hep-ph]

[26] J. R. Andersen, V. Del Duca, S. Frixione, C. R. Schmidt, and W. J. Stirling, JHEP 02, 007 (2001), hepph/0101180M. Fontannaz, J. P. Guillet, and G. Heinrich,
Eur. Phys. J. C22, 303 (2001), hep-ph/0107262

[27] P. Aurenche, R. Basu, and M. Fontannaz, Eur. Phys. J. C57, 681 (2008), arXiv:0807.2133 [hep-ph]

[28] L. H. Orr and W. J. Stirling, Phys. Rev. D56, 5875 (1997), hep-ph/9706529

[29] V. Del Duca and C. R. Schmidt, Phys. Rev. D51, 2150 (1995), hep-ph/9407359C. Marquet and C. Royon, D79, 034028 (2009), arXiv:0704.3409 [hep-ph]

[30] S. J. Brodsky and L. Di Giustino, Phys. Rev. D86, 085026 (2012), arXiv:1107.0338 [hep-ph] 5. J. Brodsky and X.-G. Wu, D85, 034038 (2012), arXiv:1111.6175 [hep-ph]Phys. Rev. Lett. 109, 042002 (2012), arXiv:1203.5312 [hep-ph]Phys. Rev. D85, 114040 (2012), arXiv:1205.1232 [hep-ph].M. Mojaza, S. J. Brodsky, and X.-G. Wu, Phys. Rev. Lett. 110, 192001 (2013), arXiv:1212.0049 [hep-ph]X.-G. Wu, S. J. Brodsky, and M. Mojaza, Prog. Part. Nucl. Phys. 72, 44 (2013), arXiv:1302.0599 [hep-ph]5. J. Brodsky, M. Mojaza, and X.-G. Wu(2013), arXiv:1304.4631 [hep-ph] X.-C. Zheng, X.-G. Wu, S.-Q. Wang, J.-M. Shen, and Q.-L. Zhang JHEP10, 117 (2013), arXiv:1308.2381 [hep-ph] 